\title{
Changes in topographical relation between the ductus arteriosus and left subclavian artery in human embryos: a study using serial sagittal sections
}

\author{
Z.W. Jin', M. Yamamoto², J.H. Kim³ ${ }^{3}$, G. Murakami ${ }^{4}$, J. Wilting ${ }^{5}$, J.F. Rodríguez-Vázquez ${ }^{6}$ \\ ${ }^{1}$ Department of Anatomy, Wuxi School of Medicine, Jiangnan University, Wuxi, China \\ ${ }^{2}$ Department of Anatomy, Tokyo Dental College, Tokyo, Japan \\ ${ }^{3}$ Department of Anatomy, Chonbuk National University Medical School, Jeonju, Republic of Korea \\ ${ }^{4}$ Division of Internal Medicine, Sapporo Asuka Hospital, Sapporo, Japan \\ 5Institute of Anatomy and Cell Biology, School of Medicine, Georg-August-Universität Göttingen, Germany \\ ${ }^{6}$ Department of Anatomy and Human Embryology, Institute of Embryology, Faculty of Medicine, \\ Complutense University, Madrid, Spain
}

[Received: 4 December 2018; Accepted: 18 February 2019]

Background: At birth, the ductus arteriosus (DA) merges with the aortic arch in the slightly caudal side of the origin of the left subclavian artery (SCA). Since the SCAs ( $7^{\text {th }}$ segmental arteries) were fixed on the level of the $7^{\text {th }}$ cervical-first thoracic vertebral bodies, the confluence of DA should migrate caudally. We aimed to describe timing and sequence of the topographical change using serial sagittal sections of 36 human embryos and foetuses (CRL 8-64 mm; 5-10 weeks), Those made easy evaluation of the vertebral levels possible in a few section.

Materials and methods: The DA or $6^{\text {th }}$ pharyngeal arch artery seemed to slide down in front of the sympathetic nerve trunk along 1.0-1.2 $\mathrm{mm}$ from the second cervical vertebral level at 5-6 weeks and, at 6 weeks (CRL 14-17 mm), the DA confluence with aorta reached the $7^{\text {th }}$ cervical level. Because of the highly elongated common carotid artery, the sliding of DA confluence seemed to be much shorter than the cervical vertebrae growing from $1 \mathrm{~mm}$ to $2.4 \mathrm{~mm}$.

Results: At the final topographical change at 6-7 weeks, the DA confluence further descended to a site 1-vertebral length below the left SCA origin. From 6 to 9 weeks, a distance from the top of the aortic arch to the left SCA origin was almost stable: $0.3-0.5 \mathrm{~mm}$ at 6 weeks and $0.4-0.6 \mathrm{~mm}$ at 9 weeks.

Conclusions: The heart descent and the caudal extension of the trachea and bronchi, those occurred before the DA sliding, were likely to be a major driving force for the sliding. (Folia Morphol 2019; 78, 4: 720-728)

Key words: heart descent, ductus arteriosus, subclavian artery, topographical anatomy, pharyngeal arch, human embryo

\section{INTRODUCTION}

At birth, the ductus arteriosus (DA) merges with the aortic arch in the caudal side of the origin of the left subclavian artery (SCA). However, in em- bryos, the heart and DA start their development in the craniocaudal level of the larynx, and later they descend together into the future thorax $[5,12]$. The aortic arch (or the DA) originates from the $4^{\text {th }}$ (or $\left.6^{\text {th }}\right)$

Address for correspondence: Dr. Z.W. Jin, Department of Anatomy, Wuxi School of Medicine, Jiangnan University, 1800 Lihu Avenue, Wuxi, Jiangsu, 214122, China, tel: +86-510-8519-7079, fax: +86-510-8519-3570, e-mail: zwjin@ybu.edu.cn 
pharyngeal arch artery. According to our studies on the pharyngeal arch structures $[1,6,7,13]$, the initial vertebral levels of these arteries almost correspond to the second-third vertebral bodies. At the site, the larynx, parathyroid and thymus together develop from the pharyngeal pouches. In contrast, the SCA or the $7^{\text {th }}$ intersegmental artery originates from a site far caudal to the pharyngeal arch arteries. Thus the initial SCA as well as the union of the left and right embryonic aortic arches is distant from the confluence of the DA with aorta. The SCA seems to be fixed by the limb bud and its associated lower cervical nerves [3].

Recent excellent studies demonstrated details of sequential changes in the arterial pole of the embryonic heart $[2,11]$. However, the vertebral level of the DA confluence was not identified. According to excellent three-dimensional (3D) reconstructions by Rana et al. [10], an $1.5 \mathrm{~mm}$ distance from the cranially-located top of the aortic arch to the caudally-located SCA origin at Carnegie stage 18 (crown-rump length [CRL] $15-20 \mathrm{~mm}$ ) is stable and maintained even in adults. Is it an illusion that the DA confluence migrates caudally along the aortic arch? Otherwise, before the stage of CRL 15-20 mm, does the DA confluence really migrate caudally? As demonstrated by Fukuoka et al. [4] who studied kidney ascent, a measurement on a single sagittal section seemed to be more accurate than a computer-aided 3D reconstruction of amount of horizontal sections. Consequently, using sagittal sections of human embryos and foetuses, we aimed to describe and measure the topographical change of the aorta, DA, left SCA and cervical vertebrae.

\section{MATERIALS AND METHODS}

This study was performed in accordance with the provisions of the Declaration of Helsinki 1995 (as revised in 2013). We observed serial sagittal sections of 36 human embryos and foetuses at 5-10 weeks (CRL 8-64 mm). Twenty-six specimens were belonging to Blechschmidt collection in Medical Museum of Georg-August-Universität Götingen (the collection, introduced by Radlanski and Renz [9]) and serial sagittal sections had been prepared. Most sections were stained with haematoxylin and eosin (H\&E), while a minor part with azan or Masson trichrome staining. The use of this collection did not require a specific approval of the Institute. The other ten specimens were a part of the large collection kept at the Embryology Institute of the Universidad Complutense, Madrid, and were the products of miscarriages and ectopic pregnancies at the Department of Obstetrics of the University. Most sections were stained with $\mathrm{H \& E}$, while a minor part with azan, orange $\mathrm{G}$ or silver staining. The use of the specimens was approved by University Ethics Committee (B08/374). Observations and taking photographs were usually performed with Nikon Eclipse 80 .

\section{RESULTS}

\section{Topographical changes of the great arteries}

In the smallest group of the present specimens (CRL 8-9 $\mathrm{mm} ; 5$ weeks), the $4^{\text {th }}$ and $6^{\text {th }}$ pharyngeal arteries (future aortic arch and DA) sandwiched a distinct pharyngeal pouch (Fig. 1). These arteries were located at the vertebral level of the second cervical vertebra. The heart base had already reached the mid-thoracic vertebral level and it was followed by the growth of the trachea and bronchi (Table 1, Fig. 1). Along the dorsal aspect of the aorta (strictly, the primitive aortic arch in the left side), the left SCA was identified as a thick segmental artery near the $7^{\text {th }}$ cervical vertebra (Fig. 1C, E). The developing sympathetic nerve trunk was attached to the vertebral column and it was separated from the aorta by a belt-like loose tissue of $0.05-0.1 \mathrm{~mm}$ in width (Fig. 1A, B).

In specimens of 13-13.5 mm CRL, although they were still at 5 weeks, a final morphology of the aortic arch appeared in close relation with the tracheal bifurcation descended to the mid-thoracic level (Table 1, Fig. 2). Thus, a confluence of DA with the aortic arch was clearly identified (Fig. 2B, D). A distance in the vertebral level between the DA confluence and left SCA origin varied between specimens, i.e., a high DA confluence 1.5 vertebral length above the origin of the left SCA (Fig. 2E) vs. a low DA near the origin of SCA (Fig. 2A).

In specimens of 14-17 mm CRL, the DA confluence consistently reached the vertebral level of the $7^{\text {th }}$ cervical or first thoracic vertebra that was almost same as the left SCA origin (Table 1, Fig. 3). In particular, one of two specimens of $17 \mathrm{~mm}$ CRL carried the left SCA originating from the same vertebral level as the DA confluence (Fig. 3G, H). In the later stages, the clavicle and sternum appeared and the thymus reached the pericardial sac (Fig. 4). The left SCA origin was consistently located $1-1.5$ vertebral length above the DA confluence: the distance was as same as (Fig. 4A) or greater than (Fig. 4D) the thickness of the DA or aorta. The topographical change in the vertebral level of the DA and left SCA was graphically demonstrated in Figure 5. 


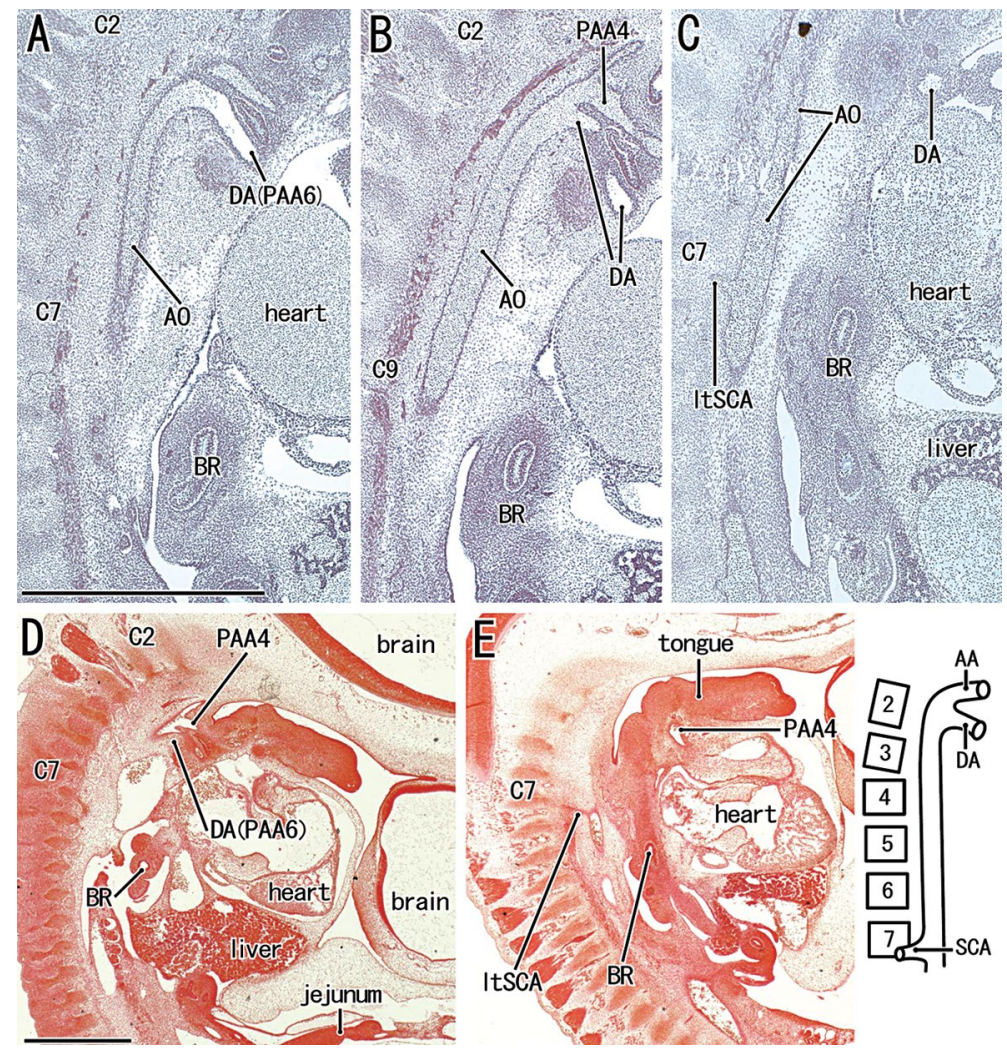

Figure 1. A-E. Sagittal sections of the aortic arch and ductus arteriosus at 5 weeks. Panels A-C display a specimen of $11 \mathrm{~mm}$ crown-rump lenght (CRL) and panels $D$ and $E$ a specimen of $8.9 \mathrm{~mm}$ CRL. Panels $A$ and $D$ exhibit the most medial site in the specimen. In both specimens, the future aortic arch (PAA4) and ductus arteriosus (PAA6, DA) are located at the level of the second cervical vertebra (C2). The left subclavian artery (ItSCA) is identified as a segmental artery thicker than the others.

Panels $A-C$ (or $D$ and $E$ ) were prepared at the same magnification (scale bar in panels $A$ and $D, 1 \mathrm{~mm}$ ). An insert at the lower angle shows a summary of the figure. Common abbreviations: $A A-$ parts of the aortic arch; $\mathrm{AO}$ - dorsal aorta; BR - left principal bronchus; $\mathrm{C} 2$ or $\mathrm{C} 7$, the second or seventh cervical vertebra; CCA - common carotid artery; $\mathrm{CRL}$ — crown-rump length; DA — ductus arteriosus; ES - oesophagus; PA — pulmonary artery; PAA4 or PAA6 - fourth or sixth pharyngeal arch artery; SCA — subclavian artery; T2 — the second thoracic vertebra.

\section{Measurements in sagittal sections}

A data of distances between great arteries as well as the vertebral size is shown in Table 2 and the major results are summarized in Figure 6 . In contrast to gradual increase of the vertebral size, the distance between the future aortic arch and the left subclavian arterial origin decreased from $0.8-1.2 \mathrm{~mm}$ to $0.3-$ $-0.5 \mathrm{~mm}$ at 5-6 weeks. In specimens of CRL 8-9 mm (5 weeks), the future aortic arch was $0.1 \mathrm{~mm}$ above the DA, which corresponded to 1 vertebral length (a height of the corpus vertebrae) in the same embryo (Fig. 6). At this initial stage, the left SCA origin was located 1.0-1.2 mm lower than the second cervical vertebra behind the future aortic arc and DA. At 6 weeks, a considerable elongation of the common carotid artery compensated a rapid growth of the vertebral column: $2.0-2.4 \mathrm{~mm}$ from the second to the $7^{\text {th }}$ cervical vertebrae (Fig. 6). In contrast, a distance between the DA and the top of the aortic arch was maintained within a height of one cervical vertebra: i.e., $0.2-0.4 \mathrm{~mm}$ at 6 weeks and $0.5-1.0 \mathrm{~mm}$ at 9 weeks (Fig. 6). In all specimens examined (5-10 weeks), one vertebral length or height gradually increased 10 times or more $(0.1 \mathrm{~mm}$ to $1.0-1.2 \mathrm{~mm})$.

After the DA confluence reached the level of the left SCA origin in a stage of $17 \mathrm{~mm} \mathrm{CRL} \mathrm{(Fig.} 3 \mathrm{H}$ ), later, a distance between the DA confluence and SCA origin increased from $0.4 \mathrm{~mm}$ (6 weeks) to $1 \mathrm{~mm}$ (9 weeks), i.e., $0.5-1.5$ vertebral length ( $1 \mathrm{~mm}$ or more at 9 weeks). Because the vertebra grew constantly, the distance between the DA confluence and SCA origin was continuously increased. However, a distance from the top of the aortic arch (the $4^{\text {th }}$ pharyngeal artery) to the left SCA ranged from 1.0-1.2 $\mathrm{mm}$ in specimens of 8-9 mm CRL and, in specimens of CRL 14-17 mm, the top of the aortic arch was still located 1.0-1.5 mm above the left SCA origin. A distance from the aortic arch to the left SCA was rather smaller $(0.3-0.6 \mathrm{~mm})$ at 6-9 weeks. A thickness of the aorta and DA increased from $0.1 \mathrm{~mm}$ at 5 weeks, via $0.2 \mathrm{~mm}$ at 6 weeks, to $1.0-1.5 \mathrm{~mm}$ at 9-10 weeks.

Consequently, the topographical relation between the vertebra and DA confluence drastically changed at 5-6 weeks. Thus, the DA confluence as well as the aortic arch was most likely to slide caudally in front of the sympathetic nerve trunk. A fixation of the DA and aortic arch by cardiac nerves was seen after 6 weeks. However, a length of the greatly growing vertebral column was largely compensated by growth of the common carotid artery. After the DA confluence reached the vertebral level of the left SCA origin at 6 weeks, the topographical relation among the DA, 
Table 1. Vertebral levels of the left subclavian arterial origin, a confluence of the ductus arteriosus, the heart base and tracheal bifurcation

\begin{tabular}{|c|c|c|c|c|c|c|}
\hline Specimen & CRL [mm] & Left SCA & DA & Heart base & TB & Comparative vertebral height \\
\hline $7-7-54$ & 8.1 & $\mathrm{C7}$ & $\mathrm{C2}$ & $\mathrm{T} 2$ & $\mathrm{~T} 1$ & -5 \\
\hline $8-6-52$ & 8.9 & $\mathrm{C7}$ & C2 & $\mathrm{T} 4 / 5$ & T3 & -5 \\
\hline $4-4-51$ & 9.0 & $\mathrm{C7}$ & $\mathrm{C} 2$ & T4 & $\mathrm{T} 3$ & -5 \\
\hline $20-7-46$ & 10.0 & $\mathrm{C7}$ & C4 & $\mathrm{T} 4$ & $\mathrm{~T} 2$ & -3 \\
\hline IW5 & 11.0 & $\mathrm{C7}$ & C2 & $\mathrm{T} 3$ & $\mathrm{~T} 2$ & -5 \\
\hline $7-3-54$ & 11.0 & $\mathrm{C7}$ & C3 & T4 & T3 & -4 \\
\hline $19-1-59$ & 13.0 & $\mathrm{C} 7 / \mathrm{T} 1$ & $\mathrm{C} 6 / 7$ & T5 & T4 & -1 \\
\hline $18-5-49$ & 13.0 & $\mathrm{C7}$ & $\mathrm{C} 6 / 7$ & $\mathrm{~T} 3 / 4$ & $\mathrm{~T} 4$ & -0.5 \\
\hline $5-1-66$ & 13.4 & $\mathrm{C7}$ & C5 & $\mathrm{T} 3 / 4$ & $\mathrm{~T} 2$ & -2 \\
\hline $8-12-50$ & 13.5 & $\mathrm{C7}$ & $\mathrm{C} 5 / 6$ & $\mathrm{~T} 5$ & $\mathrm{~T} 4$ & -1.5 \\
\hline $19-6-47$ & 14.0 & C7 & C6 & T4 & $\mathrm{T} 3 / 4$ & -1 \\
\hline 23-9-58 & 14.9 & $\mathrm{~T} 1$ & $\mathrm{C7}$ & $\mathrm{T} 4$ & $\mathrm{~T} 4$ & -1 \\
\hline $2-1-68$ & 15.0 & C7 & C6 & $\mathrm{T} 4 / 5$ & T3 & -1 \\
\hline IW16 & 15.0 & $\mathrm{C} 7 / \mathrm{T} 1$ & $\mathrm{C7}$ & $\mathrm{T} 4$ & $\mathrm{~T} 3$ & -0.5 \\
\hline GV3 & 16.0 & $\mathrm{C7}$ & $\mathrm{C} 7 / \mathrm{T} 1$ & $\mathrm{T7}$ & $\mathrm{T} 4 / 5$ & 0.5 \\
\hline $17-4-52$ & 16.8 & $\mathrm{C} 7 / \mathrm{T} 1$ & $\mathrm{~T} 1$ & $\mathrm{~T} 4 / 5$ & T4 & 0.5 \\
\hline IW33 & 17.0 & $\mathrm{C7}$ & $\mathrm{C} 6 / 7$ & $\mathrm{~T} 4$ & $\mathrm{~T} 4$ & -0.5 \\
\hline $18-3-48$ & 17.0 & $\mathrm{C7}$ & C7 & T6 & $\mathrm{T} 4 / 5$ & 0 \\
\hline $13-7-49$ & 21.0 & $\mathrm{C} 7 / \mathrm{T} 1$ & $\mathrm{~T} 1 / 2$ & T5 & $\mathrm{T} 4$ & 1 \\
\hline $22-4-52$ & 22.2 & $\mathrm{~T} 1$ & $\mathrm{~T} 2 / 3$ & $\mathrm{~T} 5 / 6$ & $\mathrm{~T} 3$ & 1.5 \\
\hline $24-6-49$ & 26.0 & $\mathrm{~T} 1$ & $\mathrm{~T} 2$ & T6 & $\mathrm{T} 4$ & 1 \\
\hline IW111 & 28.0 & $\mathrm{~T} 1$ & $\mathrm{~T} 2$ & T5 & T3 & 1 \\
\hline IW40 & 28.0 & $\mathrm{C} 7 / \mathrm{T} 1$ & $\mathrm{~T} 1 / 2$ & T5 & $\mathrm{T} 4$ & 1 \\
\hline $15-12-39$ & 29.0 & $\mathrm{~T} 1$ & $\mathrm{~T} 2 / 3$ & $T 5 / 6$ & T4 & 1.5 \\
\hline $25-6-48$ & 30.0 & $\mathrm{C} 7 / \mathrm{T} 1$ & $\mathrm{~T} 2 / 3$ & T5 & $\mathrm{T} 5$ & 2 \\
\hline $27-6-49$ & 30.5 & $\mathrm{~T} 1$ & $\mathrm{~T} 2 / 3$ & $T 5 / 6$ & T4 & 1.5 \\
\hline $27-8-51$ & 31.0 & $\mathrm{C} 7 / \mathrm{T} 1$ & T2 & T6 & $\mathrm{T} 4$ & 1.5 \\
\hline IW32 & 35.0 & C6 & $\mathrm{C7/T1}$ & $\mathrm{T} 5$ & T3 & 1.5 \\
\hline $2-6-49$ & 35.0 & $\mathrm{~T} 1 / 2$ & T3 & $\mathrm{T} 6 / 7$ & T4 & 1.5 \\
\hline IW-K36 & 36.0 & $\mathrm{~T} 1$ & $\mathrm{~T} 2$ & $\mathrm{T7}$ & T4 & 1 \\
\hline $17-12-48$ & 41.0 & $\mathrm{~T} 1 / 2$ & $\mathrm{~T} 2 / 3$ & T6 & T4 & 1 \\
\hline 23-4-50 & 45.0 & $\mathrm{~T} 1$ & $\mathrm{~T} 2$ & T6 & $\mathrm{T} 3 / 4$ & 1 \\
\hline IW-H46 & 46.0 & $\mathrm{~T} 1$ & $\mathrm{~T} 2$ & $\mathrm{~T} 7$ & T4 & 1 \\
\hline $8-6-49$ & 51.0 & $\mathrm{~T} 2 / 3$ & $\mathrm{~T} 4$ & $\mathrm{~T} 6 / 7$ & $\mathrm{~T} / 4 / 5$ & 1.5 \\
\hline IW-C56 & 56.0 & $\mathrm{~T} 1$ & $\mathrm{~T} 2$ & $\mathrm{~T} 7$ & T4 & 1 \\
\hline $24-4-50$ & 64.0 & $\mathrm{~T} 2$ & T3 & $\mathrm{T} 7$ & T4 & 1 \\
\hline
\end{tabular}

$\mathrm{CRL}$ — crown-rump length [mm]; left SCA — left subclavian arterial origin; DA — confluence of the ductus arteriosus with aorta; TB — tracheal bifurcation; comparative vertebral height, a vertebral length from the DA confluence to the left SCA origin (minus distance, the DA confluence is higher than left SCA origin). $\mathrm{C}$ - cervical vertebral body; $\mathrm{T}$ — thoracic vertebral body

SCA, aortic arch and vertebra changed a little. Nevertheless, depending on a further growth of the vertebral column, a growth of the great arteries should continue.

\section{DISCUSSION}

In spite of classical detailed descriptions of early development of the arterial pole of the human heart
$[8,14,15]$, strangely, we had no morphometrical information about a topographical change between the DA and left SCA. The present study demonstrated morphometrically a sliding of the aortic arch and DA confluence at 5-6 weeks. Since the cervical sympathetic nerve trunk, including segmental ganglia, appeared to be established at and until 5 weeks, the 

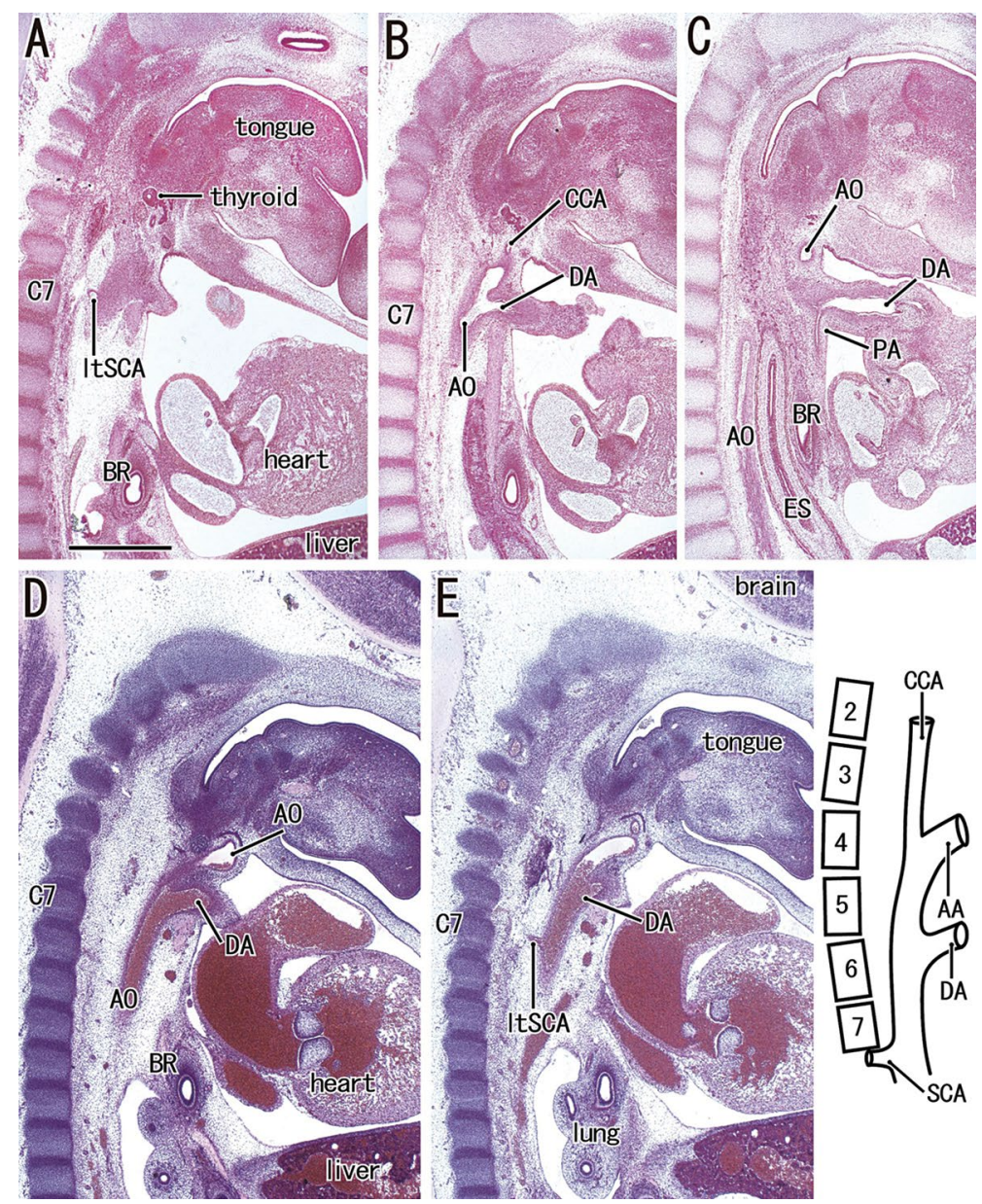

Figure 2. A-E. Sagittal sections of the aortic arch and ductus arteriosus at 5-6 weeks. Panels A-C display a specimen of $13 \mathrm{~mm} C R L$, while panels $D$ and $E$ show a specimen of $13.5 \mathrm{~mm}$ CRL. Panels $C$ and $D$ exhibit the most medial site in the specimen. In panels $\mathrm{A}-\mathrm{C}$, a confluence of the ductus arteriosus (DA) is located at the level between the six and seventh cervical vertebrae (C7) that is 0.5 vertebral length above the origin of the left subclavian artery (ItSCA). However, in panels $D$ and $E$, the confluence is 1.5 vertebral lengths above the origin. All panels were prepared at the same magnification (scale bar in panel A, $1 \mathrm{~mm}$ ). An insert at the lower angle shows a summary of the figure. Other abbreviations - see the list of common abbreviations - Fig. 1.
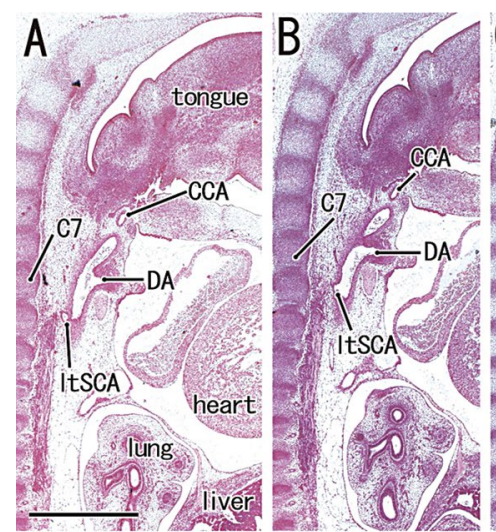

$\mathrm{F}$
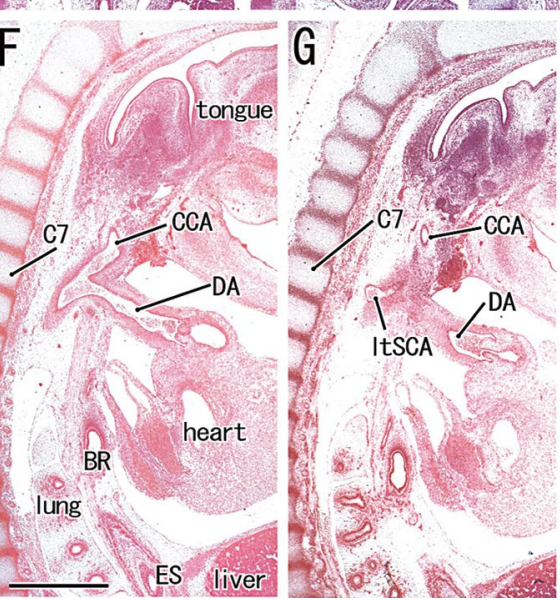

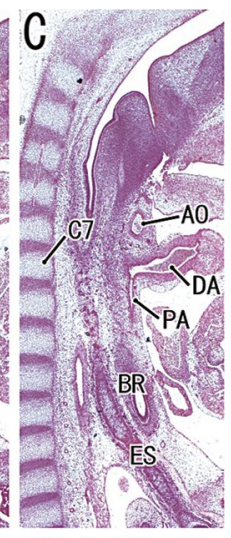

$\mathrm{H}$

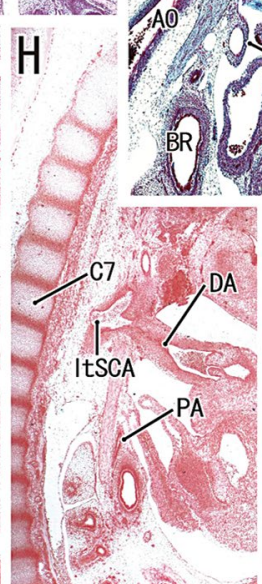

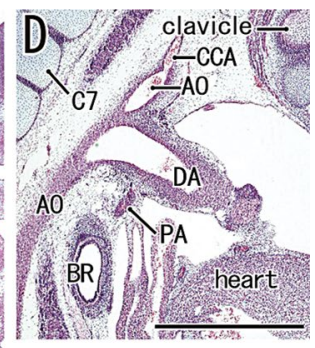

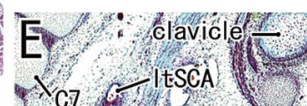

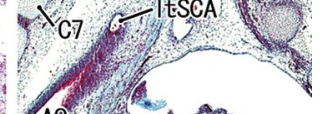

Figure 3. A-H. Sagittal sections of the aortic arch and ductus arteriosus of three specimens at 6 weeks. Panels A-C display a specimen of $14.9 \mathrm{~mm}$ CRL, while panels $D$ and $E$ show a specimen of $16 \mathrm{~mm}$ CRL. Panels F-H exhibit a specimen of $17 \mathrm{~mm}$ CRL. Panels $C, D$ and $F$ show the most medial site in the specimen. The confluence of the ductus arteriosus (DA) is located 1 vertebral length above the origin of the left subclavian artery (ItSCA) in panel $A, 0.5$ vertebral length below the origin in panels $D$ and $E$ and, at the same vertebral level in panels F-H. All panels were prepared at the same magnification (scale bar in panel A, $1 \mathrm{~mm}$ ). An insert at the lower angle shows a summary of the figure. Other abbreviations - see the list of common abbreviations - Fig. 1. 

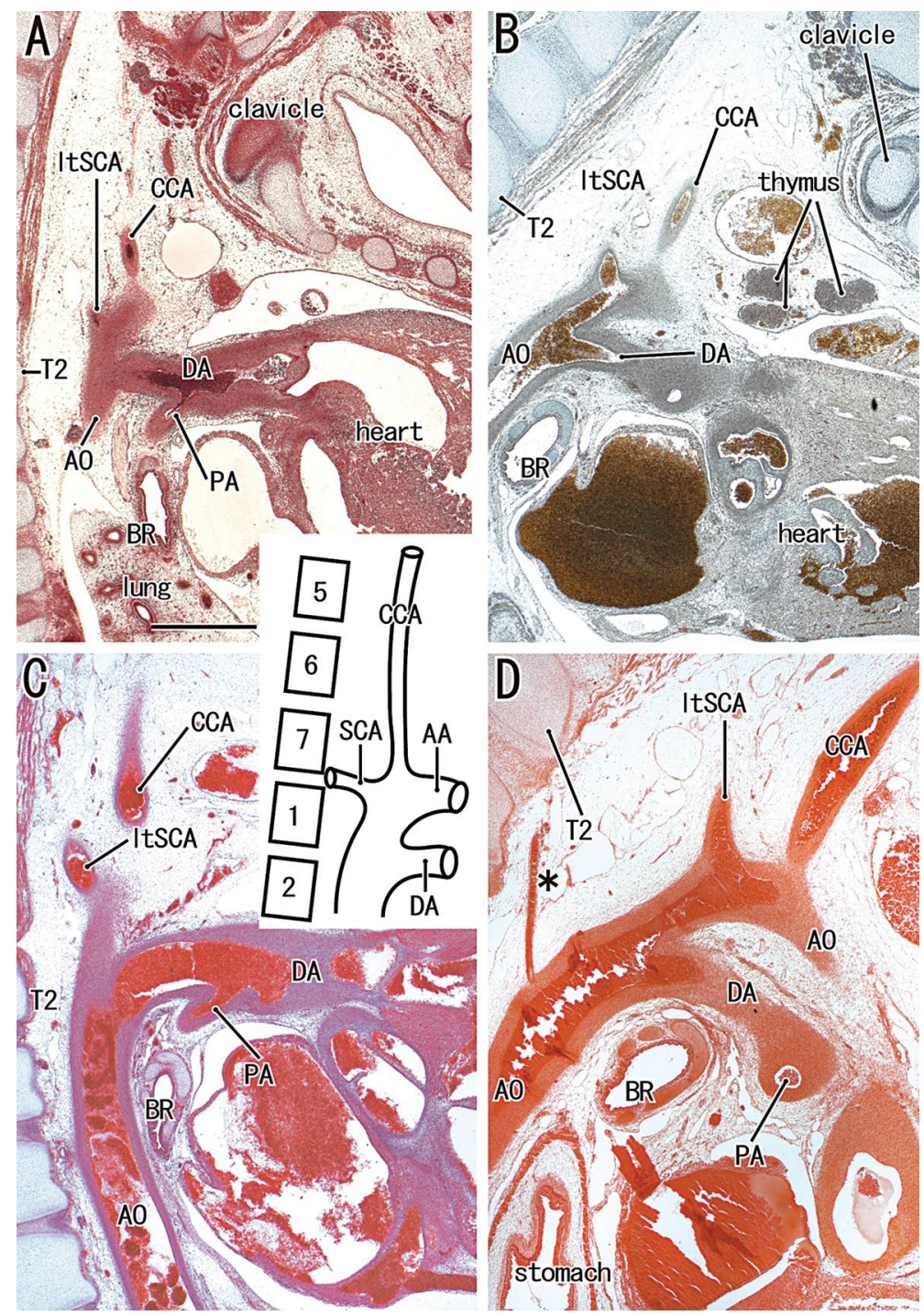

Figure 4. A-D. Sagittal sections of the aortic arch and ductus arteriosus of four specimens at 7-9 weeks. Panel A-D display a specimens of $14.9 \mathrm{~mm}$ $\mathrm{CRL}$, that of $35 \mathrm{~mm} \mathrm{CRL}$, that of $45 \mathrm{~mm} \mathrm{CRL}$ and that of $51 \mathrm{~mm}$ CRL, respectively. In these panels, the origin of the left subclavian artery (ItSCA) is located 1-1.5 vertebral length above the confluence of the ductus arteriosus (DA). Asterisk in panel $D$ indicates the second intercostal artery. The bony thorax is developed. All panels were prepared at the same magnification (scale bar in panel $A, 1 \mathrm{~mm}$ ). An insert at the lower angle shows a summary of the figure. Other abbreviations - see the list of common abbreviations - Fig. 1. descent or sliding seemed to occur in front of the nerves. The descent of DA was not an imagination product from a hypothetical difference in growth rate between the posterior and anterior aspects of the aortic arch but an actual moving along the vertebral column. An initial distance from the DA to the left SCA origin (1.0-1.2 mm) decreased to $0.3-0.5 \mathrm{~mm}$ possibly due to a rapid descent of the heart at 5-6 weeks. Surprisingly, this decreased distance did not recover until 10 weeks in spite of the 10-times growth in vertebral length. The cervical vertebrae increased almost $1.4 \mathrm{~mm}$ in length at 5-6 weeks and almost $1.2 \mathrm{~mm}$ at 6-9 weeks (total $2.6 \mathrm{~mm}$ ). When we considered the decreased length of the DA-SCA distance $(0.5-0.9 \mathrm{~mm})$, the sliding distance of the DA seemed to be estimated as $1.5-1.9 \mathrm{~mm}$. Almost all of this length seemed to be compensated by an upward elongation of the common carotid artery.
The heart descent as well as a descent of the trachea and bronchi started before the sliding of DA. In contrast, a change of the curvature of the cervical-thoracic vertebral column was not distinct during the sliding. After the initial and long sliding of the DA to reach the level of the left SCA, both topographical relations were reversed at 6-7 weeks: the DA confluence became one-vertebral length (1-1.2 mm) lower than the SCA origin. This final descent of DA might require traction by the lung expanding caudally. A short-cut course from the DA to the descending aorta seemed to provide an increased blood flow through the DA, and this would likely accelerate the final descent of the DA along one vertebral segment. Because of the development much later than the heart descent, a contribution of developing bony thorax seemed to be very limited for the topographical change of the DA confluence. 


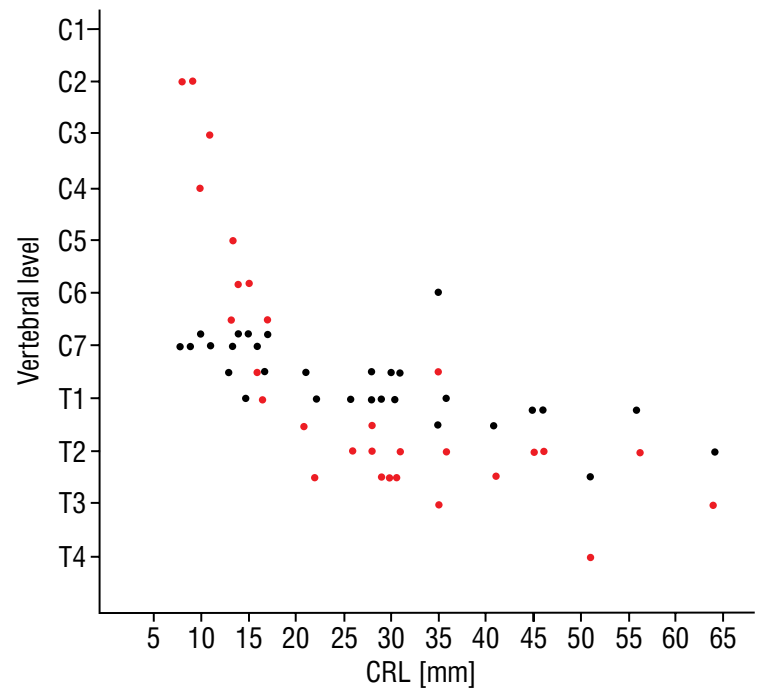

Figure 5. Graphic demonstration of the vertebral height of the confluence of the ductus arteriosus and the origin of the left subclavian artery. Red dots indicate the confluence of the ductus arteriosus, while black dots line the origin of the left subclavian artery. $\mathrm{X}$ axis corresponds to the crown-rump length (CRL, $\mathrm{mm}$ ), while $Y$ axis the vertebral level ( $\mathrm{C}$ - cervical; $\mathrm{T}$ - thoracic). Thirty-six specimens (Table 1) are plotted. In specimens of 15-17 mm CRL, the ductus arteriosus descends lower than the left subclavian artery.
Rana et al. [10] recently emphasized that a distance between the confluence of the DA and the top of the aortic arch is stable $(1.5 \mathrm{~mm})$ from Carnegie stage 18 (15-20 mm CRL) to even adults. This $1.5 \mathrm{~mm}$ measured in their 3D reconstruction was greater than our measurement in a single sagittal section $(0.3-0.6 \mathrm{~mm}$ at 6-9 weeks). Reduction in size during histological procedure seemed not be so great as the difference between our data and Rana et al. [10]. However, we agreed with a fact that the distance was almost stable. As they suggested, cell proliferation and growth seemed to be very limited at a part of the aortic wall between the left SCA origin and the top of the aortic arch.

\section{Limitations of the study}

Sagittal sections had a great advantage to evaluate the topographical change along the craniocaudal axis. However, in them, a bilateral identification of the aortic arches and pharyngeal arch arteries was difficult. According to our previous study using both sagittal and horizontal sections [1], the right aortic arch regresses

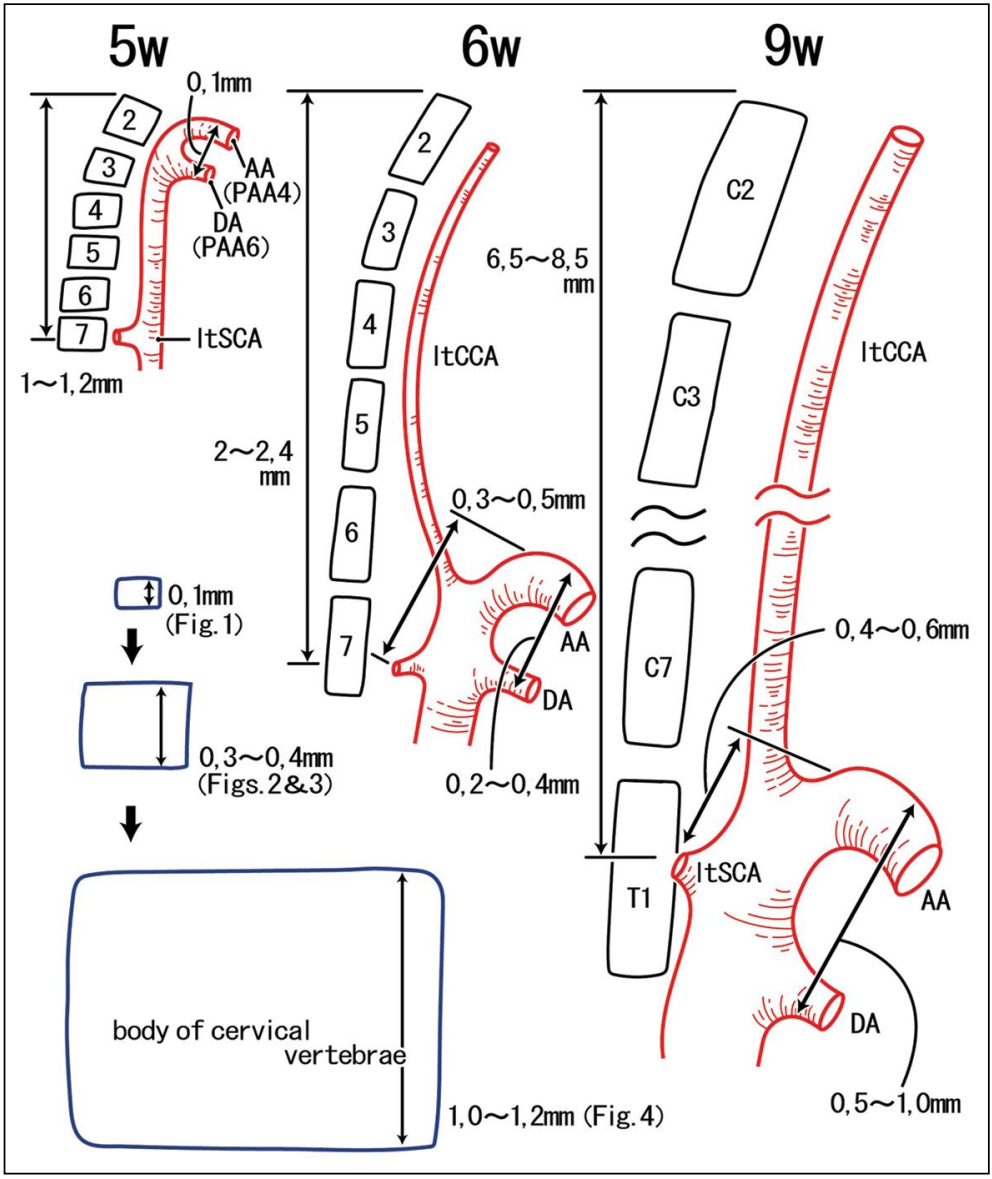

Figure 6. Schematic representation showing a topographical change of the vertebral level of the aortic arch (AA), ductus arteriosus and left subclavian artery (ItSCA). From 5 weeks to 9 weeks, a length from the second to sixth cervical vertebra increases $1 \mathrm{~mm}$ to $8.5 \mathrm{~mm}$ at maximum. Likewise, a distance between the top of the $A A$ and the ductus arteriosus (DA) also increases 10-times at maximum. In contrast, a change in a distance from the AA to the origin of the ItSCA is less than 2-times ( $0.3 \mathrm{~mm}$ to $0.6 \mathrm{~mm}$ at maximum) between 6 and 9 weeks. At 6 weeks, the confluence of the DA is located at the almost same vertebral level as the origin of the ItSCA. Three squares in the left lower side exhibit growth of a cervical vertebral body from 5 weeks to 9 weeks: the height increases 10-times. Other abbreviations - see the list of common abbreviations - Fig. 1. 
Table 2. Measurements of the distances between arteries as well as the size of cervical vertebrae

\begin{tabular}{|c|c|c|c|c|}
\hline Specimen & CRL [mm] & AA-SCA & AA-DA & Vertebral size \\
\hline $7-7-54$ & 8.1 & 1.0 & 0.1 & 0.1 \\
\hline $8-6-52$ & 8.9 & 1.0 & 0.1 & 0.1 \\
\hline $4-4-51$ & 9.0 & 1.2 & 0.1 & 0.1 \\
\hline $20-7-46$ & 10.0 & 1.1 & 0.1 & 0.1 \\
\hline IW5 & 11.0 & 0.8 & 0.1 & 0.1 \\
\hline $7-3-54$ & 11.0 & 1.0 & 0.1 & 0.1 \\
\hline $19-1-59$ & 13.0 & 0.8 & 0.2 & 0.2 \\
\hline $18-5-49$ & 13.0 & 0.3 & 0.2 & 0.3 \\
\hline $5-1-66$ & 13.4 & 0.7 & 0.2 & 0.2 \\
\hline $8-12-50$ & 13.5 & 0.4 & 0.4 & 0.3 \\
\hline $19-6-47$ & 14.0 & 0.5 & 0.3 & 0.3 \\
\hline $23-9-58$ & 14.9 & 0.5 & 0.4 & 0.3 \\
\hline $2-1-68$ & 15.0 & 0.4 & 0.4 & 0.4 \\
\hline IW16 & 15.0 & 0.5 & 0.3 & 0.4 \\
\hline GV3 & 16.0 & 0.3 & 0.4 & 0.3 \\
\hline $17-4-52$ & 16.8 & 0.4 & 0.3 & 0.4 \\
\hline IW33 & 17.0 & 0.5 & 0.4 & 0.5 \\
\hline $18-3-48$ & 17.0 & 0.5 & 0.4 & 0.5 \\
\hline $13-7-49$ & 21.0 & 0.4 & 0.4 & 0.4 \\
\hline $22-4-52$ & 22.2 & 0.5 & 0.5 & 0.6 \\
\hline $24-6-49$ & 26.0 & 0.6 & 0.8 & 0.7 \\
\hline IW111 & 28.0 & 0.4 & 0.7 & 0.8 \\
\hline IW40 & 28.0 & 0.6 & 0.8 & 0.6 \\
\hline $15-12-39$ & 29.0 & 0.5 & 0.7 & 0.9 \\
\hline $25-6-48$ & 30.0 & 0.6 & 0.6 & 0.8 \\
\hline $27-6-49$ & 30.5 & 0.4 & 0.6 & 1.0 \\
\hline $27-8-51$ & 31.0 & 0.4 & 0.8 & 1.0 \\
\hline IW32 & 35.0 & 0.5 & 0.8 & 1.0 \\
\hline $2-6-49$ & 35.0 & 0.6 & 0.8 & 0.9 \\
\hline IW-K36 & 36.0 & 0.6 & 0.8 & 1.1 \\
\hline $17-12-48$ & 41.0 & 0.6 & 1.0 & 1.0 \\
\hline $23-4-50$ & 45.0 & 0.7 & 1.2 & 1.0 \\
\hline IW-H46 & 46.0 & 0.6 & 0.9 & 1.0 \\
\hline $8-6-49$ & 51.0 & 0.7 & 1.0 & 1.0 \\
\hline IW-C56 & 56.0 & 0.7 & 1.0 & 1.2 \\
\hline $24-4-50$ & 64.0 & 0.8 & 1.1 & 1.2 \\
\hline
\end{tabular}

$\mathrm{CRL}$ - crown-rump length (mm); AA-SCA — a distance between the left subclavian arterial origin and the top of the aortic arch; AA-DA — a distance between the aortic arch and ductus arteriosus; vertebral size - a height of the seventh cervical vertebral

at a stage for the great sliding of the DA and right aortic arch at 5-6 weeks. Likewise, sagittal sections had a disadvantage to show the aortic sac including its horn that changes into the common carotid artery as well as the proximal segment of the aortic arch.

\section{CONCLUSIONS}

However, as the vertebral column and bony thorax increase in size, the aortic arch itself should grow after Carnegie stage 18 to the late-stage near birth. 


\section{Acknowledgements}

This work was supported by the National Natural Science Foundation of China (No. 81460471) and Six Talent Peaks Project in Jiangsu Province (SZCY-001).

\section{REFERENCES}

1. Abe $H$, Yamamoto $M$, Suzuki $R$, et al. Changes in topographical relation between the ductus arteriosus and left subclavian artery in human embryos: a study using serial sections. Okajimas Folia Anat Jpn. 2017; 94(1): 27-35, doi: 10.2535/ofaj.94.27, indexed in Pubmed: 29213016.

2. Anderson RH, Chaudhry B, Mohun TJ, et al. Normal and abnormal development of the intrapericardial arterial trunks in humans and mice. Cardiovasc Res. 2012; 95(1): 108-115, doi: 10.1093/cvr/cvs147, indexed in Pubmed: 22499773.

3. Barry A. The aortic arch derivatives in human adult. Anat Rec. 1951; 111(2): 221-238, indexed in Pubmed: 14894834.

4. Fukuoka K, Wilting J, Rodríguez-Vázquez JF, et al. The Embryonic Ascent of the Kidney Revisited. Anat Rec (Hoboken). 2019; 302(2): 278-287, doi: 10.1002/ar.23930, indexed in Pubmed: 30290083.

5. Hamilton WJ, Mossman HW. Human embryology. 4th ed. Williams \& Wilkins, London 1978: 230-271.

6. Honkura $Y$, Yamamoto $M$, Yoshimoto $T$, et al. Is the ultimobranchial body a reality or myth: a study using serial sections of human embryos. Okajimas Folia Anat Jpn. 2016; 93(2): 29-40, doi: 10.2535/ofaj.93.29, indexed in Pubmed: 27904020.

7. Jin ZW, Yamada T, Kim JiH, et al. Pathogenesis of solitary right aortic arch: a mass effect hypothesis based on observations of serial human embryonic sections. Cardiol Young.
2017; 27(2): 359-368, doi: 10.1017/S1047951115002152, indexed in Pubmed: 26435328.

8. Orts-Llorca F, Puerta Fonolla J, Sobrado J. The formation, septation and fate of the truncus arteriosus in man. J Anat. 1982; 134(Pt 1): 41-56, indexed in Pubmed: 7076544.

9. Radlanski RJ, Renz H. An atlas of prenatal development of the human orofacial region. Eur J Oral Sci. 2010; 118(4): 321-324, doi: 10.1111/j.1600-0722.2010.00756.x, indexed in Pubmed: 20662903.

10. Rana MS, Sizarov A, Christoffels VM, et al. Development of the human aortic arch system captured in an interactive three-dimensional reference model. Am J Med Genet A. 2014; 164A(6): 1372-1383, doi: 10.1002/ajmg.a.35881, indexed in Pubmed: 23613216.

11. Sizarov A, Lamers WH, Mohun TJ, et al. Three-dimensional and molecular analysis of the arterial pole of the developing human heart. J Anat. 2012; 220(4): 336-349, doi: 10.1111/j.14697580.2012.01474.x, indexed in Pubmed: 22296102.

12. Skandalakis JE, Gray SW. Embryology for surgeons. 2nd ed. Williams \& Wilkins, Baltimore 1994: 976-1030.

13. Takanashi Y, Honkura Y, Rodriguez-Vazquez JF, et al. Pyramidal lobe of the thyroid gland and the thyroglossal duct remnant: a study using human fetal sections. Ann Anat. 2015; 197: 29-37, doi: 10.1016/j.aanat.2014.09.001, indexed in Pubmed: 25458181.

14. Thompson RP, Sumida H, Abercrombie V, et al. Morphogenesis of human cardiac outflow. Anat Rec. 1985; 213(4): 578-86, 538, doi: 10.1002/ar.1092130414, indexed in Pubmed: 4083538.

15. Van Mierop LH, Alley RD, Kausel HW, et al. Pathogenesis of transposition complexes. I. Embryology of the ventricles and great arteries. Am J Cardiol. 1963; 12: 216-225, doi: 10.1016/0002-9149(63)90311-4, indexed in Pubmed: 14047494. 